EPJ Web of Conferences 37, 09004 (2012)

DOI: $10.1051 /$ epjconf/20123709004

C) Owned by the authors, published by EDP Sciences, 2012

\title{
Isospin Decomposition of the Basic Double-Pionic Fusion in the Region of the ABC Effect
}

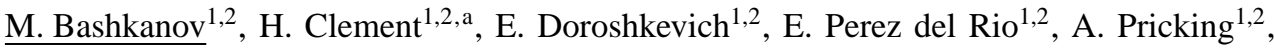

T. Skorodko ${ }^{1,2}$, and G. J. Wagner ${ }^{1,2}$ for the WASA-at-COSY Collaboration

1 Physikalisches Institut, Univ. Tübingen, Auf der Morgenstelle 14, D-72076 Tübingen, Germany

2 Kepler Center for Astro and Particle Physics, Univ. Tübingen, Auf der Morgenstelle 14, D-72076 Tübingen

\begin{abstract}
With a proton beam of $T_{p}=1.2 \mathrm{GeV}$ incident on the deuterium pellet target of the WASA detector setup all three basic double-pionic fusion reactions have been measured simultaneously. By use of quasifree kinematics the energy range $2.3 \mathrm{GeV} \leq \sqrt{s}<$ $2.5 \mathrm{GeV}$ could be covered, which just coincides with the energy region, where the $\mathrm{ABC}$ effect and its associated resonance structure has been observed. From the isospin decomposition we see that the resonance effect is solely in the isoscalar part of the reaction process, whereas the isovector part exhibits a monotonic smoothly rising energy dependence and no $\mathrm{ABC}$ effect.
\end{abstract}

\section{Introduction}

The ABC effect, an intriguing low-mass enhancement in the $\pi \pi$ invariant mass spectrum, is known from inclusive measurements of the production of an isoscalar pion pair in fusion reactions to light nuclei - first observed by Abashian, Booth and Crowe in the $p d \rightarrow{ }^{3} \mathrm{He} \mathrm{X}$ reaction [1]. Its explanation has been a puzzle since more than 50 years and hence has been named just after the initials of those authors, who first observed this effect. Together with low-statistics bubble chamber measurements these experiments suggested that this effect is correlated with the production of an isoscalar pion pair and with the fact that the participating nucleons fuse to a bound nuclear system in the final state [2-11].

Recent data on the basic double-pionic fusion reaction $p n \rightarrow d \pi^{0} \pi^{0}$ demonstrate that the so-called $\mathrm{ABC}$ effect is tightly correlated with a narrow resonance structure in the total cross section of this reaction $[12,13]$. In these exclusive and kinematically complete high-statistics measurements of the isoscalar $p n \rightarrow d \pi^{0} \pi^{0}$ reaction it has been shown that the $\mathrm{ABC}$ effect is correlated with a pronounced resonance structure in the total cross section at $\sqrt{s}=2.37 \mathrm{GeV}$ with a width of only $70 \mathrm{MeV}$ and quantum numbers $I\left(J^{P}\right)=0\left(3^{+}\right)$[12]. That means that this structure is situated about $90 \mathrm{MeV}$ below $\sqrt{s}=2 m_{\Delta}$, the peak position of the conventional $t$-channel $\Delta \Delta$ process, and has a width, which is about three times narrower than this process.

\section{Experiment and Results}

In an effort to solve this long-standing issue by exclusive and kinematically complete high-statistics experiments, we have measured all three basic fusion reactions to deuterium

$-p n \rightarrow d \pi^{0} \pi^{0}$
$-p n \rightarrow d \pi^{+} \pi^{-}$

a e-mail: clement@pit.physik.uni-tuebingen.de

This is an Open Access article distributed under the terms of the Creative Commons Attribution License 2.0, which permits unrestricted use, distribution, and reproduction in any medium, provided the original work is properly cited. 


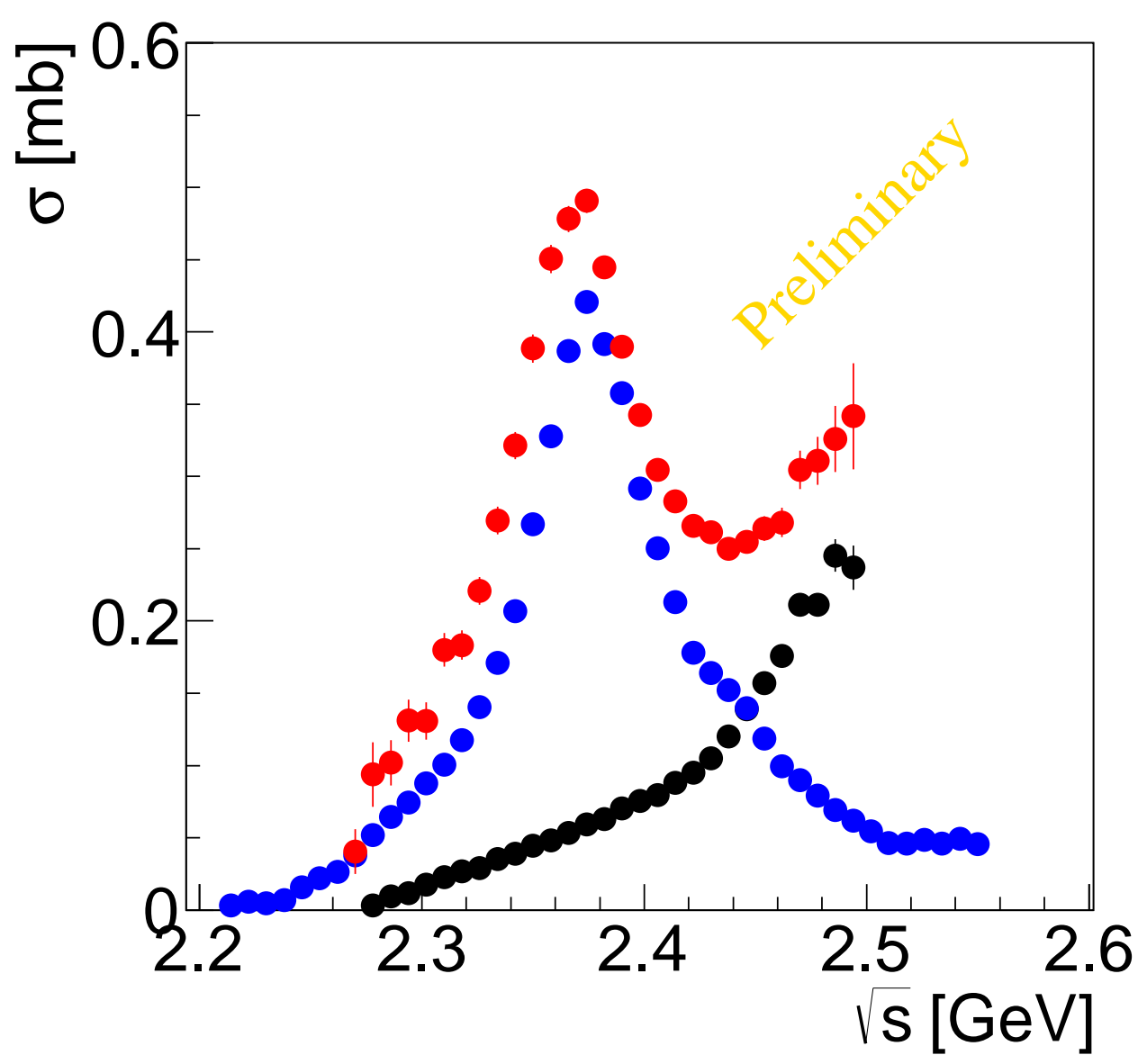

Fig. 1. Preliminary results for the total cross sections of the basic double-pionic fusion reactions $p N \rightarrow d \pi \pi$ of different isospin systems in dependence of the center-of-mass energy $\sqrt{s}$ from threshold $(\sqrt{s}=2.15 \mathrm{GeV})$ up to $2.6 \mathrm{GeV}$. The data for the isospin mixed reaction $p n \rightarrow d \pi^{+} \pi^{-}$are shown by the red full circles. The data for its isovector part given by half the cross section of the $p p \rightarrow d \pi^{+} \pi^{0}$ reaction are plotted by the black filled circles, whereas the data for its isoscalar part given by twice the cross section of the $p n \rightarrow d \pi^{0} \pi^{0}$ reaction are shown by the blue filled circles. From Ref. [17].

$$
-p p \rightarrow d \pi^{+} \pi^{0}
$$

with WASA at COSY $[14,15]$. These measurements have been carried out with a proton beam hitting the deuterium pellet target at $T_{p}=1.2 \mathrm{GeV}$ in quasifree kinematics. That way the energy range $2.3 \mathrm{GeV} \leq \sqrt{s}<02.5 \mathrm{GeV}$ has been covered, which coincides with the energy region, where the $\mathrm{ABC}$ effect has been observed previously.

The purely isovector reaction $p p \rightarrow d \pi^{+} \pi^{0}$ (Fig. 1, black filled circles) exhibits a smooth energy dependence in the total cross section, which is monotonically rising in the observed energy interval, as well as no ABC effect. This is not unexpected, since due to Bose symmetry the isovector pion pair can not be in relative $s$-wave and hence any low-mass enhancement would be suppressed in this situation. Also as shown in Ref. [16] this reaction is reasonably well described by conventional $t$-channel $\Delta \Delta$ excitation, which is known to be the leading process in all $p p$ induced, i.e. isovector two-pion production channels at beam energies above $1 \mathrm{GeV}[18,19]$. 
In contrast, the purely isoscalar fusion reaction $p n \rightarrow d \pi^{0} \pi^{0}$ does not behave as expected from conventional reaction dynamics. It rather exhibits a narrow resonance structure in the total cross section, which is correlated with the appearance of the ABC effect in the $\pi^{0} \pi^{0}$ invariant mass spectrum. The cross section maximum is about $90 \mathrm{MeV}$ below $2 m_{\Delta}$, the mass of a $\Delta \Delta$ system, and its width of only $70 \mathrm{MeV}$ is three times smaller than $2 \Gamma_{\Delta}$ expected from the conventional $t$-channel $\Delta \Delta$ process. Based on the measured angular distributions the quantum numbers $I\left(J^{P}\right)=0\left(3^{+}\right)$have been assigned to this structure [12]. At present no conventional process is known, which could at least qualitatively explain this phenomenon.

The $p n \rightarrow d \pi^{+} \pi^{-}$reaction contains both isoscalar and isovector parts. Its data are well understood by combining the results from the $p n \rightarrow d \pi^{0} \pi^{0}$ and $p p \rightarrow d \pi^{+} \pi^{0}$ reactions according to the isospin relation [20]

$$
\sigma\left(p n \rightarrow d \pi^{+} \pi^{-}\right)=2 \sigma\left(p n \rightarrow d \pi^{0} \pi^{0}\right)+\frac{1}{2} \sigma\left(p p \rightarrow d \pi^{+} \pi^{0}\right) .
$$

The data also demonstrate, why previous low-statistics bubble-chamber measurements of this reaction with neutron beams of low momentum resolution had actually no chance to discover the narrow resonance structure in the $d \pi^{+} \pi^{-}$channel.

\section{Acknowledgments}

We acknowledge valuable discussions with C. Hanhart, E. Oset, A. Sibirtsev and C. Wilkin on this issue. This work has been supported by BMBF (06TU9193) and Forschungszentrum Jülich (COSYFFE).

\section{References}

1. N. E. Booth, A. Abashian, K. M. Crowe, Phys. Rev. Lett. 7 (1961) 35; 5 (1960) 258; Phys. Rev. 132 (1963) 2296ff

2. I. Bar-Nir et al., Nucl. Phys. B 54 (1973) 17

3. A. Abdivaliev et al., Sov. J. Nucl. Phys. 29(1979) 796

4. R. J. Homer et al., Phys. Rev. Lett. 9 (1964) 72

5. J. H.Hall et al., Nucl. Phys. B 12 (1969) 573

6. J. Banaigs et al., Nucl. Phys. B 67 (1973) 1

7. J. Banaigs et al., Nucl. Phys. B 105 (1976) 52

8. F.Plouin et al., Nucl. Phys. A 302 (1978) 413

9. F. Plouin, P. Fleury, C. Wilkin, Phys. Rev. Lett. 65 (1990) 690

10. R. Wurzinger et al., Phys. Lett. B 445 (1999) 423

11. for a review see A. Codino and F. Plouin, $\mathrm{LNS} / \mathrm{Ph} / 94-06$

12. P. Adlarson et al., Phys. Rev. Lett. 106, (2011) 202302 (2011) 202302

13. M. Bashkanov et al., Phys. Rev. Lett. 102 (2009) 052301; arXiv: 0806.4942 [nucl-ex]

14. Ch. Bargholtz et al., Nucl. Instrum. Methods A 547, (2005) 294

15. H. H. Adam et al., arXiv:nucl-ex/0411038, (2004)

16. F. Kren et al., Phys. Lett. B 684, (2010) 110 and B 702 (2011) 312; arXiv:0910.0995v2 [nucl-ex]

17. COSY Annual Report highlights 2012

18. T. Skorodko et al., Phys. Lett. B 695, (2011) 115

19. T. Skorodko et al., Phys. Lett. B 679 (2009) 30; arXiv: 0906.3087 [nucl-ex]

20. J. Bystricky et al., J. Physique 48 (1987) 1901 